\title{
Letter to Editors \\ Hypothetical Laws While Dealing with Effect by Cause in Discrete Space
}

\author{
B. S. DAYA SAGAR* \\ Department of Geoengineering, Centre for Remote Sensing and Information Systems, Andhra University, \\ Visakhapatnam - 530003 , India
}

(Received 25 April 2000)

Whole issue of this letter, to say, is to deal with structure (a well defined physical system having a definite boundary) an effect by a cause, represented in terms of another microstructure with characteristic information. Why can't we visualise a systematic force as a symmetric force, being one of the possibility, with definite geometry having characteristic information such as an origin, a direction, an orientation, a size and a shape. Of course, the force, with center as an origin with symmetric from any angle to any defined opposite angle in polar space obviously symmetric also across scales, is a perfect symmetric morphological force. The degree of symmetry in the force with reference to the characteristic information can be well defined by considering occurred change in the structure that is subjected to transformation (the transformations being expansion, contraction, either globally or locally, and the cascade of these two in two directions in discrete space). Yet the transformations due to which the changes occur are unclassified precisely. To classify these trans- formations rather precisely a morphological force [1] can be considered for investigations. However, the derivation of morphological force strictly depends on the subtracted portion, from transformed to non-transformed fields, and the predefined morphological force (both in discrete " $n$ " dimensional space). The precision of the defined morphological force can be defined such that the morphological entropy should be zero while investigating the subtracted portion. The investigation is according to the pattern spectrum procedure [2]. As an example a circular type of structure has been transformed by an unknown transformation, say by forces of several physically, chemically, biologically defined types, in turn, say this structure has become circular with greater in size. In a simple way it can be said that the transformation made the structure at time " $t$ " as a bulged structure at time " $t+1$ " uniformly. This is a descriptive analysis. If one subtracts the transformed structure from non-transformed structure, and if that subtracted portion is investigated with

\footnotetext{
* Present address: Centre for Remote Imaging, Sensing, Processing (CRISP), Faculty of Science, The National University of Singapore, Lower Kent Ridge Road, Singapore-119260, e-mail: crsbsds@nus.edu.sg, bsdsagar@hotmail.com
} 
several morphological forces, one can single out only the morphological force of which the morphological entropy is zero. That circular type of morphological force contains all the other characteristic information. Yet a regular structure may become irregular, vice versa. Under such circumstances, either structure itself or the force can be heterogeneous. However, choosing out the involved morphological force from the occurred change needs to be paid attention. This induces scientist not bother about the type of force whether it is due to physical, mechanical, chemical, or biological force, acted on it but to bother about the collective force that needs to be defined in terms of morphological aspects in discrete space. Can all the physical forces be unified to define an entity called "Morphological force"?. Such a nagging and rather poorly formed idea has a justification at least to certain degree while understanding the features in a time sequential domain. Strictly speaking in geometric terms, two broadly categorised forces, namely endogenic, tectonic in nature, and exogenic, weather and climate based, have been altering the morphlologies of the earth systems.

Now mostly with the advent of new geometric concepts such as fractals and multifractal formalisms, the degree of spatio-temporal heterogeneity in the effect is being quantified precisely, yet ignoring fact is the spatio-temporal heterogeneity of the cause. With the significance of quantifying the spatio-temporal heterogeneity in this morphological force (cause) coupled with spatio-temporal heterogeneity that is quantified for the actual structure under transformation (effect), our understanding the complexity may advance a bit further. The reason is the constant increase in the spatiotemporal heterogeneity in this cause. This is possible when one can reduce the collective intensity of the cause, the force, into a simple morphological entity with justifiable characteristic information. The laws that could be visualised hypothetically from a geoscientific point of views are based on the properties of structures when subjected to force are as follows.
- A structure reaches the state of convergence during the process of continuous expansion by a force of size more than that of the structure. (e.g., bulge of lake)

- During the process of continuous contraction by a force of size of more than that of structure, the structure either disappears or disintegrates and then disappears. (dried up lake)

- A Euclidean type of structure will not undergo any change under the process of cascade of expansion contraction. However, there will be a variation in the transformed structure if any of the characteristics of force to expand is different from that to contract. (e.g., formation of Oxbow lake).

- Under the process of cascade of contraction expansion, if the cumulative force acting upon the structure is less than the size of the structure, the transformed structure is geometrically similar to the force. As long as the structure does not disintegrate during this process, if the force to contract is different from that to expand, the morphology of the resultant structure depends upon the succeeding force and the structure remaining just before it gets vanished during the subprocess of contraction. The structure disappears if the cumulative force is more than the structure.

- Under any process, when both structure and force are geometrically similar, and also the cumulative force does not dominate the structure, the transformed structure will be geometrically similar to both original structure and force. Critical point is the iteration number at which the structure reaches the state of convergence. This point depends upon the process, characteristics of force, and the original structure.

\section{References}

[1] Sagar, B. S. D., Venu, M., Gandhi, G. and Srinivas, D. (1998). Morphological description and interrelationship between force and structure: a scope to geomorphic evolution process modelling, International Journal of Remote Sensing, 19(7), 1341-1358.

[2] Maragos, P. A. (1989). Pattern spectra and multiscale shape representation, IEEE Transactions on Pattern Analysis and Machine Intelligence, 11(7), 701-716. 


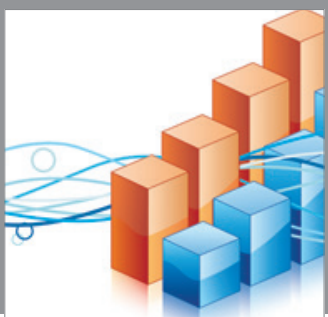

Advances in

Operations Research

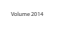

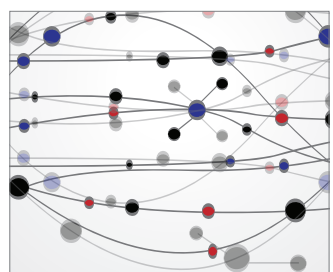

\section{The Scientific} World Journal
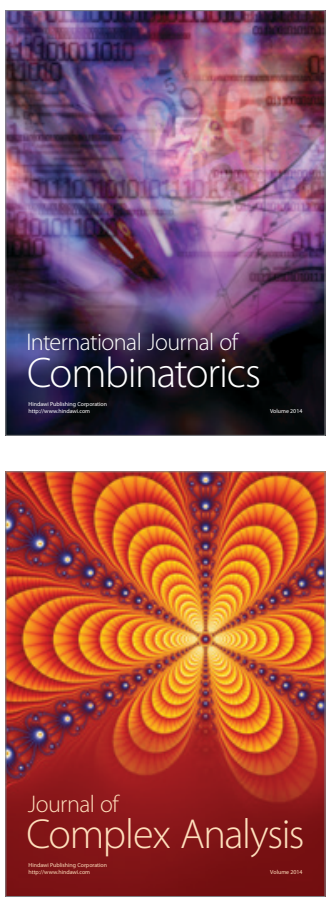

International Journal of

Mathematics and

Mathematical

Sciences
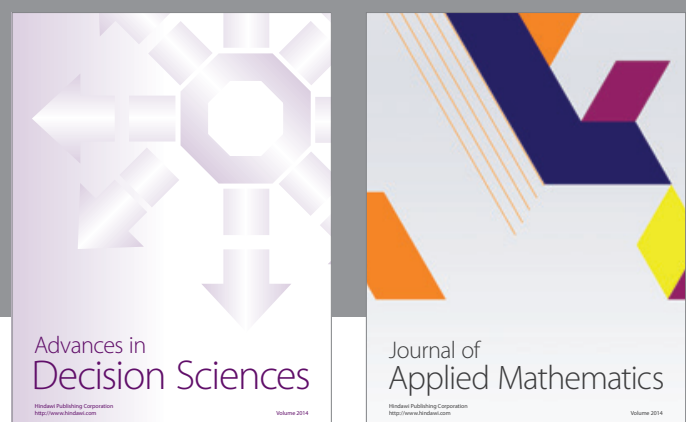

Journal of

Applied Mathematics
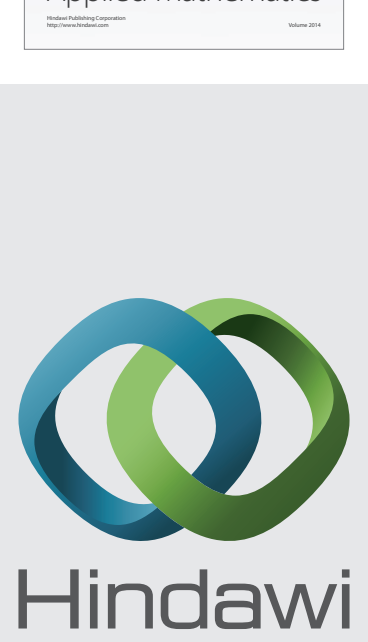

Submit your manuscripts at http://www.hindawi.com
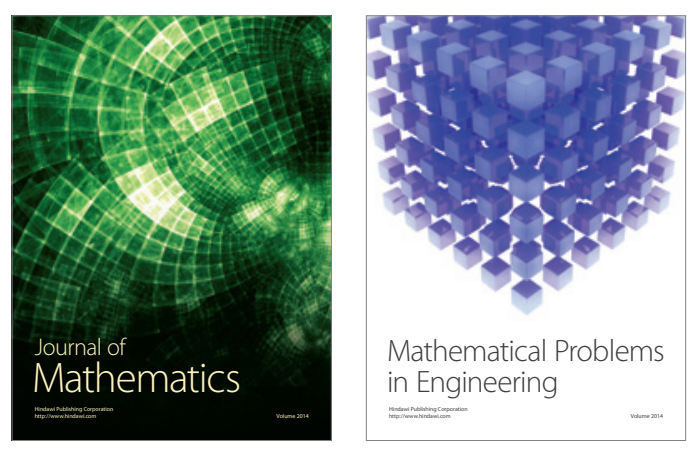

Mathematical Problems in Engineering
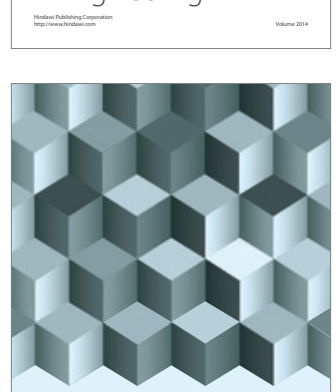

Journal of

Function Spaces
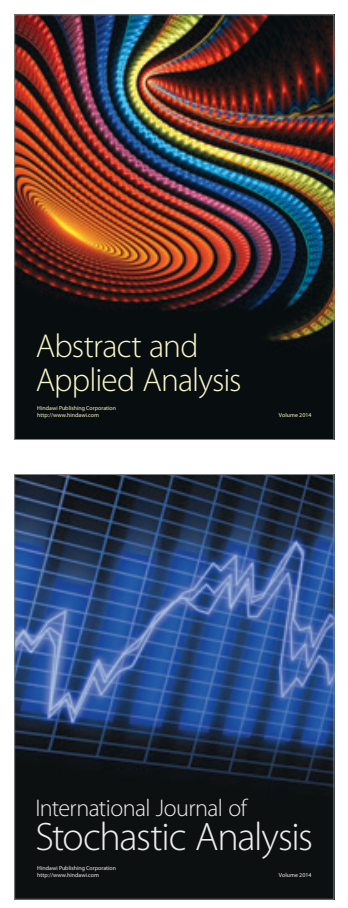

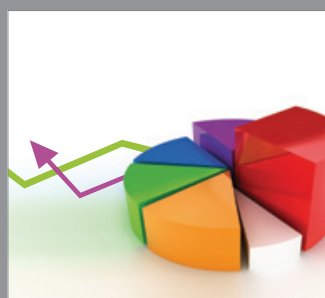

ournal of

Probability and Statistics

Promensencen
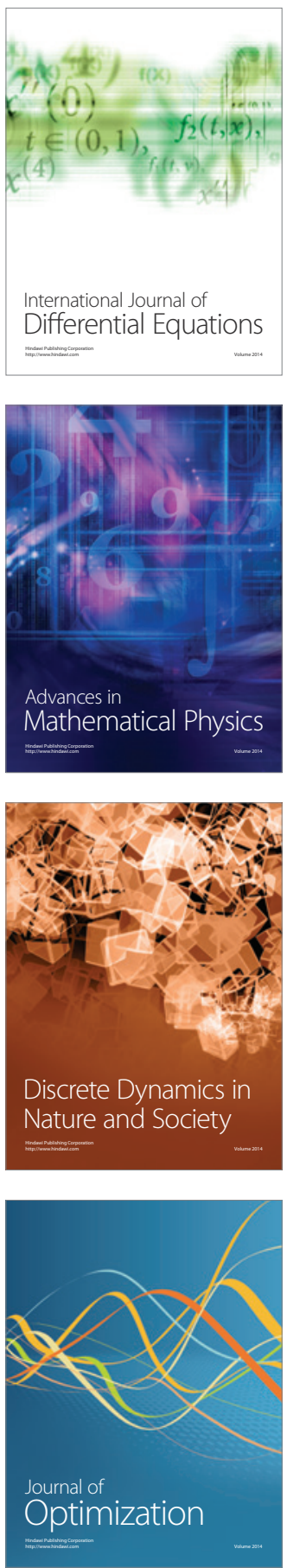